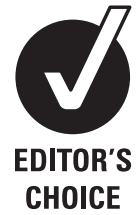

- The appendix to this paper is published online only. To view this file please visit the journal online (http://bjsm. bmj.com)

${ }^{1}$ Mayo Clinic College of Medicine, Mayo Clinic Sports Medicine Center, Rochester, Minnesota, USA 2Department of Family Medicine, Indiana University School of Medicine, South Bend, Indiana, USA; South Bend Sports Medicine Fellowship Program; AMSSM Musculoskeletal Ultrasound Curriculum

\section{Correspondence to}

Dr Mark E Lavallee, Memorial Sports Medicine Institute, 111 West Jefferson Blvd, Suite 100 South Bend, IN 46601, USA:

mlavallee@memorialsb.org

Accepted 17 September 2010

\title{
Musculoskeletal ultrasound education for sports medicine fellows: a suggested/potential curriculum by the American Medical Society for Sports Medicine
}

\author{
Jonathan Finnoff, ${ }^{1}$ Mark E Lavallee, ${ }^{2}$ Jay Smith ${ }^{1}$
}

\begin{abstract}
The following musculoskeletal ultrasound (MSK US) curriculum was developed by the American Medical Society for Sports Medicine (AMSSM) to provide a pathway by which a sports medicine fellow can obtain adequate MSK US training during their fellowship to meet the requirements of competency outlined by the American Institute of Ultrasound in Medicine (AIUM) Training Guidelines for the Performance of MSK US Examination. Many fellowship programmes may not be able to follow all of the recommendations outlined by this document owing to their available resources. However, this curriculum can be used as a suggested/potential guideline for MSK US training within a sports medicine fellowship, and may assist programmes in developing or modifying their own internal training methods.
\end{abstract}

\section{OVERVIEW}

The educational process should include four components.

\section{Didactic instructional sessions}

The first component suggested is didactic musculoskeletal ultrasound (MSK US). This can be in the form of a dedicated MSK US course or scheduled teaching within the fellowship programme (see section Introduction 252 to MSK US-Didactic Instructional Sessions). The fellow should receive instruction on basic ultrasound physics, image optimisation, advantages and limitations of MSK US, and diagnostic and interventional techniques.

\section{Didactic practice sessions}

The second aspect of the fellow's education should involve didactic sessions in which the diagnostic scanning and interventional techniques presented during the didactic instructional sessions are practised under the direct supervision of a mentor in order to become proficient with these skills (see section Diagnostic and Interventional MSK US 433-Didactic Practice Sessions).

\section{Mentored clinical experience}

Third, the fellow should perform diagnostic scanning and interventional procedures on patients in a clinical setting under the direct supervision of a mentor. Once competency for image acquisition and procedural execution has been determined, direct supervision may be curtailed as allowed by institutional policy governing teaching rules.

\section{Supplementary and continuing education}

The fourth component of the fellow's education should include supplementary education such as reading reference texts and journal articles; presenting MSK US-related articles in journal club on a regular basis; utilising MSK US-related online educational material; attending MSK US conferences and presentations; and performing independent scanning.

This natural stepwise progression of diagnostic and interventional MSK US education will ensure the acquisition of adequate MSK US skills to allow independent practice of diagnostic and interventional MSK US upon completion of their fellowship.

\section{LEARNING OBJECTIVES}

a) Identify and discuss the function of basic controls on an ultrasound machine console, including:
i) Transducer selection
ii) Depth
iii) Focal zone
iv) Gain
v) Time gain compensation/depth gain compensation
vi) Zoom

b) Discuss the basic physics principles of ultrasound, including Doppler imaging

c) Review a brief history of ultrasonic medicine

d) Demonstrate how to optimise an ultrasound image

e) Describe the normal ultrasonographic appearance of adipose, muscle, tendon, ligament, bone, fascia, vessels, nerve and cartilage

f) Discuss the benefits and limitations of MSK US

g) Identify and discuss the source and/or implications of basic ultrasound artefacts, including:
i) Anisotropy
ii) Reverberation
iii) Refraction
iv) Through transmission
v) Acoustic Shadowing

h) Perform image acquisition of vascular structures including neovessels using Colour and Power Doppler

i) Describe and perform a MSK US static and dynamic examination (including proper patient positioning) of the following regions as recommended by the American Institute of Ultrasound in Medicine (AIUM) Practice Guideline for the Performance of the MSK US Examination:
i) Shoulder
ii) Elbow 

iii) Wrist-hand
iv) Hip
v) Knee
vi) Ankle-foot

j) Obtain an acceptable set of MSK US images of the following structures (see online Appendix A):

i) Shoulder

ii) Elbow

iii) Wrist-hand

iv) Hip

v) Knee

vi) Ankle-foot

k) Demonstrate appropriate labelling of MSK US images

i) Use of text insertion

ii) Use of arrows and measurement calipers

1) Demonstrate how to capture, store and transfer MSK US images

m) Generate an appropriate MSK US report

i) Diagnostic MSK US

ii) Interventional procedures using MSK US

(1) Injections

(2) Aspirations

n) Identify and dynamically image a needle in the short axis (transverse plane or out of plane) and long axis (longitudinal plane or in plane) using MSK US guidance in a phantom, turkey breast, cadaveric specimen or other imaging medium

o) Demonstrate the ability to guide a needle into a target region or structure using a long axis and a short axis approach in a phantom, turkey breast, cadaveric specimen or other imaging medium

p) Perform the following interventional procedures:

i) Shoulder

(1) Subacromial-subdeltoid bursa injection

(2) Intra-articular glenohumeral joint injection

(3) Intra-articular acromioclavicular joint injection

(4) Bicipital tendon sheath/groove injection

ii) Elbow

(1) Intra-articular elbow joint injection

(2) Peritendinous injection of the common extensor tendon origin

(3) Peritendinous injection of the common flexor tendon origin

iii) Wrist-hand

(1) Carpal tunnel injection

(2) First dorsal compartment tendon sheath injection (ie, DeQuervain's)

(3) Intra-articular wrist injection

(4) First carpometacarpal joint injection

iv) Hip

(1) Intra-articular hip injection

(2) Greater trochanteric bursa injection

v) Knee

(1) Intra-articular knee injection

(2) Pes Anserine bursa injection

(3) Iliotibial band/bursa (distal) injection

vi) Ankle-foot

(1) Intra-articular ankle injection

(2) Peroneal tendon sheath injection

\section{RESOURCES/REFERENCES}

a) Jacobson JA. Fundamentals of Musculoskeletal Ultrasound. Philadelphia: Saunders Elsevier 2007. ISBN 978-1-41603593-0 b) Bianchi S. Ultrasound of the Musculoskeletal System. Berlin Heidelberg: Springer-Verlag 2007. ISBN 978-3-540-42267-9

c) O'Neill JMD. Musculoskeletal Ultrasound: Anatomy and Technique. New York: Springer 2008. ISBN 978-0387-76609-6

d) McNally EG. Practical Musculoskeletal Ultrasound. London: Elsevier 2005. ISBN 0-443-07350-3

e) Smith J, Finnoff JT. Diagnostic and interventional musculoskeletal ultrasound: part 1. fundamentals. $P M R$ 2009;1:64-75.

f) Smith J, Finnoff JT. Diagnostic and interventional musculoskeletal ultrasound: part 2. PMR 2009;1:162-177.

g) Musculoskeletal ultrasound scanning protocol checklist (see online Appendix A)

h) American Institute for ultrasound in medicine

i) Practice guideline for the performance of the musculoskeletal ultrasound examination

ii) Practice guideline for the performance of the shoulder ultrasound examination

iii) Training guidelines for the performance of musculoskeletal ultrasound examinations

iv) http://www.aium.org

i) European Society of skeletal radiology US scanning protocols

i) http://www.essr.org

j) American College of Radiology

i) Practice guideline for communication of diagnostic imaging findings

ii) http://www.acr.org

\section{INTRODUCTION TO MSK US—DIDACTIC INSTRUCTIONAL SESSIONS}

The introduction to MSK US didactic instructional sessions include the six basic units described in this section. Appropriate reading assignments from a reference text should be given to the fellow in preparation for each unit. Several excellent reference texts exist, many of which are listed in the Resources/ References of this document. The sample curriculum provided in this document includes reading assignments from a currently available MSK US textbook (Fundamentals of MSK US by John Jacobson), but fellowship directors may substitute reading assignments from any appropriate text. We also recommend using the MSK US scanning protocols outlined in the AIUM Practice Guideline for the Performance of the MSK US Examination when instructing the fellow on scanning techniques. A MSK US Protocol CheckList based upon this document is provided in online Appendix A.

The first unit introduces the fellow to basic MSK US physics, the history of MSK US, scanning principles, and advantages and limitations of MSK US. Units 2-5 involve demonstration by an experienced MSK ultrasonographer of the static and dynamic scanning technique(s) for one or more regions followed by practice under the direct supervision of the mentor. The fellow should only consider this an introduction to the scanning technique for each region and should frequently practice the scanning techniques independently between didactic sessions. Unit 6 involves three individual sessions dedicated to interventional MSK US procedures. Basic information regarding interventional procedures including pharmacological principles of commonly used medications, patient selection, aseptic technique with MSK US guided procedures, risks associated with interventional procedures and treatment of common adverse events associated with interventional procedures should be addressed early in unit 6 , prior to the start 
of practising the interventions. Common upper- and lowerextremity interventional procedures should be discussed, demonstrated and practised under the supervision of a mentor. Numerous mediums may be utilised for the practice of the interventional procedures. The ideal mediums are unembalmed cadaveric specimens. However, if cadaveric specimens are unavailable, the fellow can practise patient positioning and target acquisition for various interventional procedures on live models followed by practice of needle visualisation and guidance on turkey breasts, pig feet, pig legs, phantoms and/ or other non-cadaveric specimens. The fellow should practice needle visualisation and guidance techniques between mentored didactic sessions to enhance their skills.

\section{Unit 1 Principles of MSK US and an introduction to scanning techniques}

i) Required reading

(1) Jacobson pp.1-11

(2) Smith J, Finnoff JT. Diagnostic and interventional musculoskeletal ultrasound: part 1. Fundamentals. PMR 2009;1:64-75.

(3) Smith J, Finnoff JT. Diagnostic and interventional musculoskeletal ultrasound: part 2. PMR 2009;1:162-177.

ii) Introduction to MSK US and scanning

(1) Instruction in 'knobology' \& basic scanning techniques

(2) Instruction on basic US physics

(3) Review the history of diagnostic and interventional MSK US

(4) Demonstration of normal sonographic appearance of bone, cartilage, tendon, muscle, ligament, nerve and artery/vein

(5) Demonstration of the use of colour and power Doppler for imaging vascular and neo-vascular structures

(6) Demonstration of transducer movements to optimise image (translation, heel-toe, tilt, rotation, pressure/ compression)

(7) Supervised practice

\section{Unit 2 MSK US examination of the knee-hip}

iii) Reading-knee
(1) Required Jacobson
pp. $224-238$
(2) Optional Jacobson
pp. $238-263$

iv) Reading-hip \& thigh
(1) Required Jacobson
(2) Optional Jacobson
pp. $178-88$
pp. 188-93

v) Knee US scanning protocol

(1) Instruction \& supervised practice

(2) Resources

(a) MSK US Protocol Checklists-Knee

(b) AIUM Guidelines for Performance of the MSK US Examination

vi) Hip \& thigh US scanning protocol

(1) Instruction \& supervised practice

(2) Resources

(a) MSK US protocol checklists-Hip-Thigh

(b) AIUM guidelines for performance of the MSK US examination

vii) Independent scanning

Unit 3 MSK US examination of the elbow-wrist-hand viii) Reading-Elbow
(1) Required
Jacobson
pp. $102-120$
(2) Optional
Jacobson
pp. $120-132$

ix) Reading-Wrist-hand
(1) Required Jacobson
pp. 133-152
(2) Optional Jacobson

x) Elbow US scanning protocol

(1) Instruction \& Supervised Practice

(2) Resources

(a) MSK US protocol checklists-Elbow

(b) AIUM guidelines for performance of the MSK US examination

xi) Wrist-Hand US scanning protocol

(1) Instruction \& supervised practice

(2) Resources

(a) MSK US protocol checklists-Wrist-hand

(b) AIUM guidelines for performance of the MSK US examination

xii) Independent scanning

Unit 4 MSK US examination of the ankle-foot

xiii) Reading
(1) Required
Jacobson
pp. 264-287
(2) Optional
Jacobson
pp. 287-331

xiv) Ankle-foot US scanning protocol

(1) Instruction \& supervised practice

(2) Resources
(a) MSK US protocol checklists-Ankle-foot
(b) AIUM guidelines for performance of the MSK US examination

xv) Independent scanning

\section{Unit 5 MSK US examination of the shoulder}

xvi) Reading
(1) Required
Jacobson
pp. $39-53$
pp. $75-80$
(2) Optional Jacobson pp. 53-74
pp. 80-101

xvii) Shoulder US scanning protocol

(1) Instruction \& supervised practice

(2) Resources

(a) MSK US protocol checklists-shoulder

(b) AIUM guidelines for performance of the shoulder US examination

(c) AIUM guidelines for performance of the MSK US examination

xviii) Independent scanning

Unit 6 Interventional procedures using MSK US

xix) Required reading Jacobson pp.11-14

$\mathrm{xx}$ ) Lectures \& hands-on sessions

(1) Injection principles and practice

(a) Lecture and hands-on practice using phantoms, turkey breasts, pigs feet or other non-cadaveric specimens

(2) Upper extremity i

(a) Demonstration and hands-on practice using an upper extremity cadaveric specimen

i) Subacromial-subdeltoid bursa injection

ii) Intra-articular glenohumeral joint injection

iii) Intra-articular acromioclavicular joint injection

iv) Bicipital tendon sheath/groove injection

v) Intra-articular elbow joint injection

xiii) Peritendinous injection of the common extensor tendon origin 
vii) Peritendinous injection of the common flexor tendon origin

viii) Carpal tunnel injection

ix) First dorsal compartment tendon sheath injection (ie, DeQuervain's)

$x)$ Intra-articular wrist injection

xi) First carpometacarpal joint injection

(3) Lower extremity injections

(a) Demonstration and hands-on practice using a lowerextremity cadaveric specimen
i) Intra-articular hip injection
ii) Greater trochanteric bursa injection
iii) Intra-articular knee injection
iv) Pes Anserine bursa injection
v) Iliotibial band/bursa (distal) injection
vi) Intra-articular ankle injection
vii) Peroneal tendon sheath injection

\section{DIAGNOSTIC AND INTERVENTIONAL MSK US-DIDACTIC PRACTICE SESSIONS}

Didactic practice sessions should be scheduled on a regular basis especially early in training during which the fellow practices, under the direct supervision of their mentor, the diagnostic and interventional MSK US techniques introduced during the didactic instructional sessions. The didactic practice sessions should include the following:

(1) The scanning techniques for each anatomical region should be practised and relevant structures should be imaged (see the AIUM practice guideline for the performance of the MSK US examination located on the AIUM website at http://www.aium.org, and the MSK US scanning checklist provided in online Appendix A of this document).

(2) The fellow should also be instructed on proper image labelling and storage of captured images. Transference of images should follow the guidelines outlined by the Health Insurance Portability and Accountability Act (HIPAA).

(3) Between didactic practice sessions, the fellow should complete a predetermined number of regional MSK US examinations on volunteers. The images should be saved and later reviewed with the mentor to ensure they are adequate and have been labelled appropriately.

(4) Interventional procedures should be practised on a regularly scheduled basis, using cadaveric specimens, preferably unembalmed. If cadaveric specimens are not available, the fellow should practice appropriate imaging of target structures on live models, and should practice needle imaging and guidance techniques using turkey breasts, pig feet, pig legs, phantoms or other appropriate medium. As the fellow's skills improve, more advanced MSK US examination techniques and interventional procedures should be introduced into the didactic practice sessions.

\section{DIAGNOSTIC AND INTERVENTIONAL MSK US-MENTORED CLINICAL EXPERIENCE}

The sports medicine fellow should have regularly scheduled clinical time in which they receive supervised hands-on experience performing diagnostic and interventional MSK US on patients. The fellow should practise their diagnostic and interventional MSK US skills but should also focus on enhancing their image optimisation and labelling abilities, and interpretation of diagnostic MSK US examinations. It is important for the fellow to be able to communicate their findings to other healthcare professionals. It is recommended that the fellow use the American College of Radiology Practice Guideline for Communication of Diagnostic Imaging Findings to assist in formulating appropriate diagnostic and/or interventional MSK US procedure notes. Those utilising electronic medical record (EMR) may be able to develop preset reporting protocols.

This component of the sports medicine fellow's MSK US training process is imperative. It provides the fellow with a supervised environment in which to practise diagnostic and interventional MSK US skills in order to prepare them for independent practice. Fellows should be exposed to a wide variety of pathology during their diagnostic MSK US examination experiences, as well as a multitude of common interventional MSK US procedures. The fellow should be instructed on obtaining informed consent prior to performing interventional ultrasound procedures.

\section{SUPPLEMENTARY AND CONTINUING MSK US EDUCATION}

The fellow's MSK US education should not be restricted to the formal educational activities outlined in the sections Introduction 252 to MSK US-Didactic Instructional Sessions, Diagnostic and Interventional MSK US 433-Didactic Practice Sessions and Diagnostic and Interventional MSK 461 US-Mentored Clinical Experience. The number of hours to accomplish this will vary from programme to programme and from fellow to fellow. Rather, the fellow should be encouraged to:

(1) Read MSK US journals and texts on a regular basis

(2) Review MSK US related articles on regular basis. It is recommended that the fellow present a MSK US related journal article during journal club at least on a quarterly basis

(3) Participate in online MSK US related courses

(4) Read online MSK US related educational material

(5) Attend MSK US related conferences

(6) Independently practice MSK US examinations and interventional procedures on volunteers and models, respectively

(7) Consider membership to the AIUM

\section{MSK US RECORD KEEPING AND COMPETENCY}

The sports medicine fellow should maintain meticulous records of all MSK US educational activities that they participate in throughout their fellowship. In addition, the fellow should maintain a procedure log of all diagnostic and interventional MSK US procedures that they observed and performed. This information can assist when determining competency in diagnostic and interventional MSK US upon completion of their fellowship. The fellowship should attempt to meet the competency criteria outlined by the AIUM Training Guidelines for the Performance of MSK US Examinations. The portion of this document that applies to a sports medicine fellow provides the following competency guideline:

Completion of a residency or fellowship program supervised by a physician qualified to perform musculoskeletal ultrasound that provides structured musculoskeletal ultrasound training, including the performance, interpretation, and reporting of 150 musculoskeletal ultrasound examinations. Unless within 2 years of completion of a residency and/or fellowship, the physician will also need to have completed 


\section{Original article}

40 hours of AMA PRA Category 1 Credits specific to MSK ultrasound, including at least 1 MSK ultrasound course that includes hands-on training.

In addition to completing the fellowship's MSK US curriculum and meeting the minimum requirements outlined by the AIUM training guidelines (see above), it is recommended that the fellowship director/ teaching faculty develop an objective system of measurement (eg, practicum and/or written test) to determine the fellow's knowledge and skill in diagnostic and interventional MSK US and establish competency.

Competing interests None.

Provenance and peer review Not commissioned; externally peer reviewed. 